\title{
Performance Envelope and Physiological Tremor in Microsurgery
}

\author{
D. Ortega Ibáñez ${ }^{1}$, F. Perez Baquerín ${ }^{1}$, D. Y. Choi ${ }^{2}$, and C. N. Riviere ${ }^{2}$ \\ ${ }^{1}$ School of Engineering, University of Valladolid, Valladolid, Spain \\ ${ }^{2}$ The Robotics Institute, Carnegie Mellon University, Pittsburgh, PA, USA
}

\begin{abstract}
Using an instrumented surgical tool, high-precision recordings of hand tremor were taken during vitreoretinal microsurgery in a rabbit model in vivo. The data obtained using a compact, custom six-degree-of-freedom inertial sensing module were filtered and analyzed to characterize the physiological hand tremor of the surgeon. Tremor during the most delicate part of the procedure was measured at a vector magnitude of $30 \mu \mathrm{m}$ rms. Non-tremulous, lower-frequency components of instrument movement were also characterized. The data collected provide an important baseline for design specification and performance evaluation of microsurgical devices, using a more comprehensive data set than has been evaluated previously.
\end{abstract}

\section{INTRODUCTION}

Inaccuracy in manipulation is a well-known problem in microsurgery. Physiological tremor is the most familiar component of erroneous motion, though it is not the only significant component. Several types of engineered accuracyenhancement devices have been or are being developed in order to improve the manipulation accuracy of the microsurgeon, including telerobotic systems [1], the "steady-hand" robotic system [2], and a handheld micromanipulator with active tremor canceling [3]. To properly specify designs of such systems and preserve the normal control bandwidth of the surgeon, there is a need for high-quality measurements of the unassisted performance of microsurgeons. In addition, basic information on tremor amplitude under regular microsurgical conditions is needed in order to provide a baseline for evaluation of engineered devices. To date there has been a lack of tracking instruments that feature both threedimensional (3-D) sensing and the requisite level of precision, and such as do exist have been laboratory instruments that are incompatible with intraoperative use [4]. Therefore, to date such data have been generally unavailable in the literature.

This paper presents experimental results obtained during retinal microsurgical procedures in rabbits in vivo using an instrument equipped with inertial motion and magnetic sensors. The study was conducted using a completely redesigned motion-sensing instrument with higher accuracy than previous studies [5]. The results presented include an examination of the "performance envelope" used by the surgeon in terms of acceleration and velocity, and the amplitude of tremor measured during the procedures.

\section{METHODS}

Vitreoretinal microsurgery was performed in rabbits at the Doheny Eye Institute of the University of Southern California. Tests were performed in a total of five eyes in three rabbits. Tasks performed included membrane peeling, vessel puncture, and vessel dissection. Roughly 73 minutes of data were recorded.

Funding provided by National Science Foundation (grant no. EEC-9731748) and National Institutes of Health (grant no. R01 EB000526).
Motion during microsurgery was recorded using the inertial measurement unit described in [6]. The unit incorporates three miniature Analog Devices ADXL-202E dual-axis accelerometers (dimensions: $5 \mathrm{~mm} \times 5 \mathrm{~mm} \times 2 \mathrm{~mm}$, weight: $<1 \mathrm{~g}$ ). The proximal end of the instrument handle houses two dualaxis accelerometers in orthogonal orientations (with one sensor oriented along the $x$ axis, one along the $y$ axis, and two along $z$ (the long axis of the instrument). The third dual-axis accelerometer is located at the distal end close to the intraocular shaft, measuring motion in $x$ and $y$. The instrument tip has a standard Luer connector to which a needle was affixed during operation.

Acceleration data were digitized at $100 \mathrm{~Hz}$ using 16-bit precision. Rigid body kinematics were used to compute the motion at the tip of the needle using the sensor readings. Velocity and displacement estimates were obtained by integration of the acceleration data.

To obtain a tremor estimate, and a characterization of the voluntary (or at least non-tremulous) acceleration and velocity used by the surgeon, the data were then filtered as follows.

Physiological tremor. Displacement data were obtained by double integration of the acceleration. The displacement signal was then filtered with an eighth-order bandpass filter with corner frequencies at 5 and $15 \mathrm{~Hz}$ in order to isolate the tremor signal.

Performance envelope. Tip velocity was estimated by integration of the acceleration signal. Both velocity and acceleration signals were highpass filtered ( $6^{\text {th }}$ order, $0.1 \mathrm{~Hz}$ cutoff) in order to suppress the effects of integration drift and gravity. Both signals were then lowpass filtered ( $6^{\text {th }}$ order Butterworth) with a corner frequency of $5 \mathrm{~Hz}$, suppressing the physiological tremor, in order to extract the non-tremulous components of the motion. The resulting acceleration and velocity signals were then sorted in order of magnitude in order to facilitate visualization of how much of the time the signal magnitude remains below a given level.

\section{RESULTS}

Fig. 1. shows the amplitude spectral density of the acceleration using the vector norm. The results show a rounded peak between 5 and $15 \mathrm{~Hz}$. This pattern is consistent throughout the data. This led to the selection of filter corner frequencies of 5 and $15 \mathrm{~Hz}$ for this data set, as described in $\$ I I$.

The results of the tremor estimation are displayed in Figs. 2 and 3. The tremor amplitude was found to be $16 \mu \mathrm{m}, 2 \mu \mathrm{m}$, and $24 \mu \mathrm{m} \mathrm{rms}$ along the $x, y$, and $z$ axes, respectively. The vector magnitude of these rms amplitudes is $29 \mu \mathrm{m}$. If the tremor signals along each axis were perfect sinusoids, this would be equivalent to a peak-to-peak vector magnitude of 82 $\mu \mathrm{m}$. 


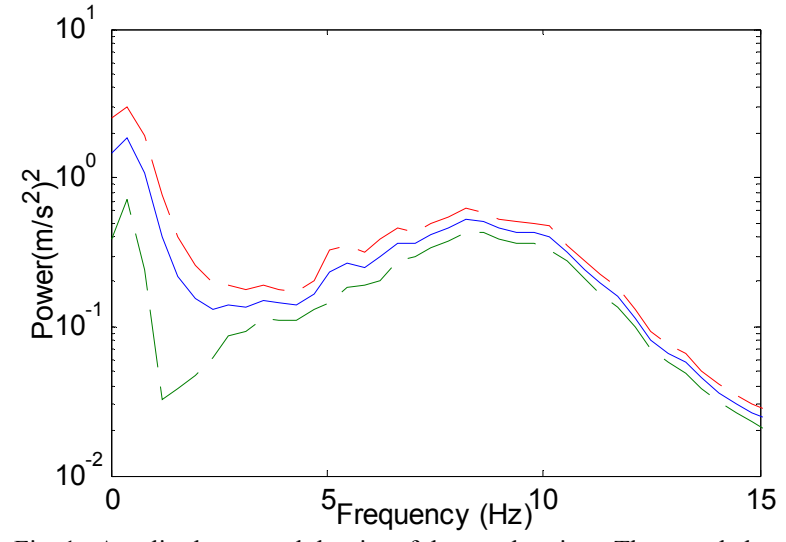

Fig. 1. Amplitude spectral density of the acceleration. The rounded peak between 5 and $15 \mathrm{~Hz}$ is consistent with what is expected due to the physiological tremor.

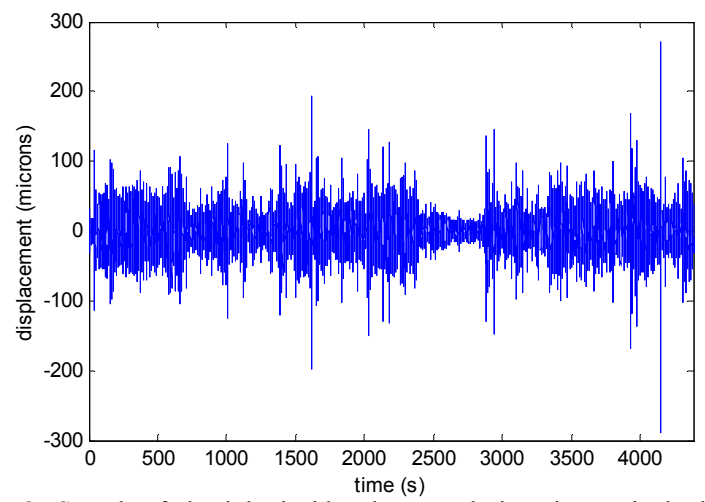

Fig. 2. Sample of physiological hand tremor during vitreoretinal microsurgery. $X$ axis.

\section{DISCUSSION AND CONCLUSIONS}

The data reported here reflect a higher-precision measuring instrument, a greater number of subjects, and a wider variety of vitreoretinal microsurgical tasks than results that have appeared previously [5]. The membrane peeling task performed here is similar in nature to epiretinal membrane peel procedures that are frequently performed. The vessel puncture task is comparable to the manipulation that is necessary to perform retinal vein cannulation. The dissection task is similar to the tissue manipulation that would be involved in arteriovenous sheathotomy. Membrane peeling is a frequently performed clinical procedure today, and both retinal vessel cannulation and arteriovenous sheathotomy are innovative procedures that are expected to become clinically relevant in the near future. As such these results are expected to be useful in the development of future microsurgical accuracy-enhancement systems by providing baseline data on performance of surgeons for use in design specification and device evaluation.

\section{REFERENCES}

[1] H. Das, H. Zak, J. Johnson, J. Crouch, D. Frambach, "Evaluation of a telerobotic system to assist surgeons in microsurgery," Computer Aided Surg,. vol. 4, pp. 15-25, 1999.

[2] R. Taylor et al., "A steady-hand robotic system for microsurgical augmentation," Int. J. Robot. Res., vol. 18, pp. 1201-1210, 1999.

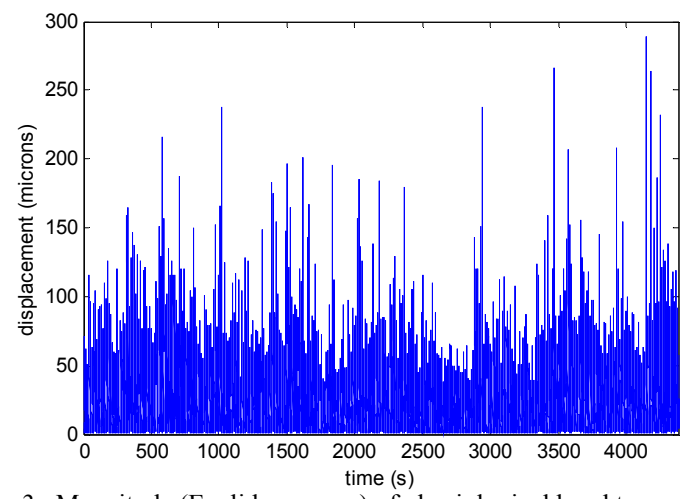

Fig. 3. Magnitude (Euclidean norm) of physiological hand tremor during vitreoretinal microsurgery.

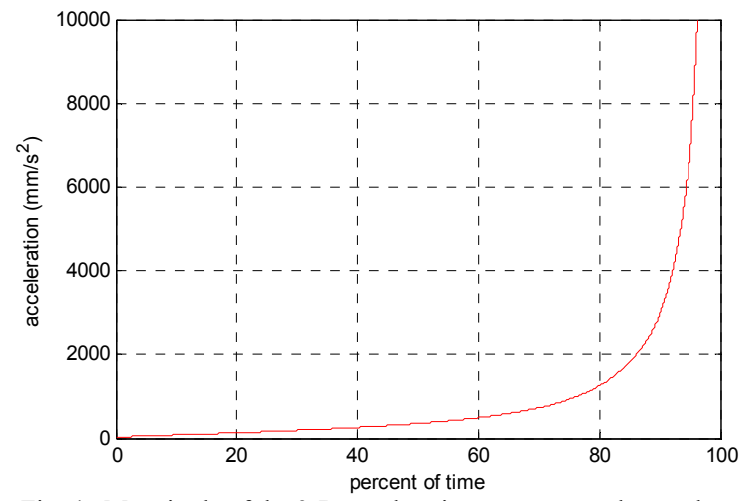

Fig. 4. Magnitude of the 3-D acceleration vector at each sample, sorted over the 73 minutes of analyzed data.

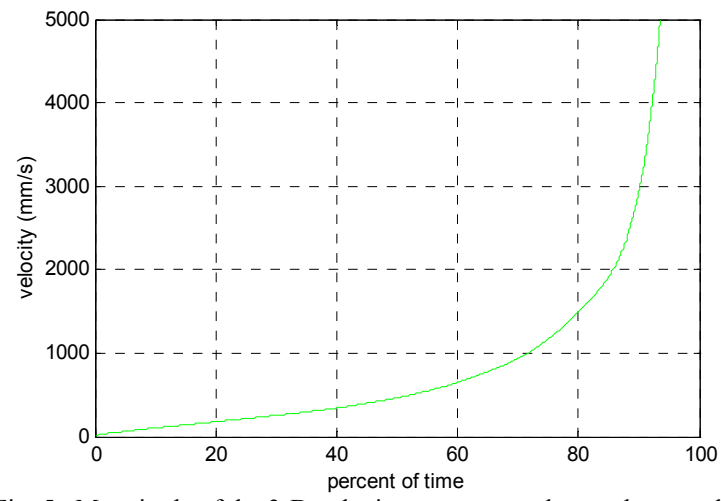

Fig. 5. Magnitude of the 3-D velocity vector at each sample, sorted over the 73 minutes of analyzed data.

[3] C. N. Riviere, W. T. Ang, and P. K. Khosla, "Toward active tremor canceling in handheld microsurgical instruments," IEEE Trans. Robot. Autom., vol. 19, pp. 793-800, 2003.

[4] L. Hotraphinyo and C. N. Riviere, "Precision measurement for microsurgical instrument evaluation,” Proc. $22^{\text {nd }}$ Intl. Conf. IEEE Eng. Med. Biol. Soc., Istanbul, Oct. 25-28, 2001, pp. 3454-3457.

[5] S. P. N. Singh and C. N. Riviere, "Physiological tremor amplitude during retinal microsurgery," Proc. $28^{\text {th }}$ Northeast Bioeng. Conf., Philadelphia, April 20-21, 2002, pp. 171-172.

[6] W. T. Ang, P. K. Khosla, and C. N. Riviere, "Design of allaccelerometer inertial measurement unit for tremor sensing in handheld microsurgical instrument," Proc. IEEE Intl. Conf. Robot. Autom., Taipei, Taiwan, Sept. 14-19, 2003, pp. 1781-1786. 